Case Report

\title{
Partial Recovery of Audiological, Vestibular, and Radiological Findings following Spontaneous Intralabyrinthine Haemorrhage
}

\author{
Thomas Pézier, ${ }^{1}$ Krisztina Baráth, ${ }^{2}$ and Stefan Hegemann" \\ ${ }^{1}$ Interdisciplinary Centre for Vertigo and Balance Disorders, ORL Klinik, University Hospital Zurich, \\ Frauenklinikstrasse 24, 8091 Zurich, Switzerland \\ ${ }^{2}$ Medical Radiological Institute Zurich, Toblerstrasse 51, 8044 Zurich, Switzerland
}

Correspondence should be addressed to Thomas Pézier; tfrpezier@gmail.com

Received 26 August 2013; Accepted 5 November 2013

Academic Editors: K. Chang, M. T. Kalcioglu, A. Rapoport, and G. Zhou

Copyright (C) 2013 Thomas Pézier et al. This is an open access article distributed under the Creative Commons Attribution License, which permits unrestricted use, distribution, and reproduction in any medium, provided the original work is properly cited.

\begin{abstract}
The diagnosis, work-up, and treatment of sudden sensorineural hearing loss and sudden vestibular loss vary widely between units. With the increasing access to both magnetic resonance imaging and objective vestibular testing, our understanding of the various aetiologies at hand is increasing. Despite this, the therapeutic options are limited and without a particularly strong evidence base. We present a rare, yet increasingly diagnosed, case of intralabyrinthine haemorrhage (ILH) together with radiological, audiological, and vestibular test results. Of note, this occurred spontaneously and has shown partial recovery in all the mentioned modalities.
\end{abstract}

\section{Introduction}

Sudden sensorineural hearing loss (SSNHL) was first described by de Kleyn in 1944 [1]. Today, it is defined as a sensori-neural hearing loss of $>30 \mathrm{~dB}$ across at least 3 consecutive frequencies arising in $<72$ hours $[2,3]$. It is generally unilateral and reports of its annual incidence vary from 5 to $300 / 100,000[4,5]$. Up to $90 \%$ of cases are idiopathic [6] and treatment is generally with steroids, though the evidence supporting this is controversial [7-9] especially as spontaneous improvement occurs in $>50 \%$ of patients within 2 weeks $[6,10,11]$. The specific type of steroid, its dose/duration, and method of delivery vary widely from unit to unit. Our unit uses very high dose oral dexamethasone and we have recently published encouraging results [12]

Intralabyrinthine haemorrhage (ILH) as a cause of SSNHL is extremely rare, and there have been few such reports in the literature [13-18]. The relationship of vestibular symptoms with spontaneous ILH is also unclear. Generally, haemorrhage occurs in patients with coagulopathies as seen in leukaemia or with anticoagulant medication and very few patients enjoy any recovery of function.

\section{Case Report}

A 50-year-old journalist presented to the ENT department at the University Hospital Zurich, with left-sided tinnitus, hearing loss, and general dizziness without real vertigo. The symptoms had suddenly appeared whilst at work a month previously and he had been treated by a private ENT doctor with oral prednisolone (50 $\mathrm{mg}$ daily for 10 days), betahistine, magnesium, and flunarizine with a diagnosis of an acute idiopathic vestibulocochlear loss. During this time his hearing loss had remained, but his dizziness symptoms had almost completely resolved. He also complained of tinnitus and a pressure sensation on the left ear. There was no further medical history of note other than that he took $100 \mathrm{mg}$ aspirin daily because of borderline increased hematocrit. He occasionally drank alcohol and was an exsmoker.

Otoscopy showed normal ear drums on both sides. There was no spontaneous or gaze evoked nystagmus. A clinical head impulse test was unclear as the patient continuously blinked. Head-shaking test showed nystagmus to the right. Pure tone audiogram (Figure 1) showed a clear asymmetry of his hearing. 


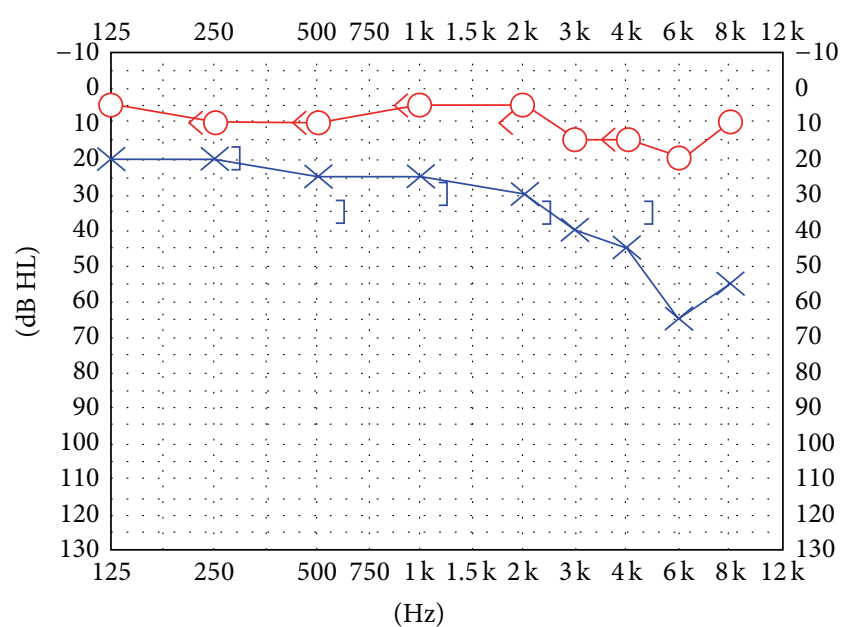

(a)

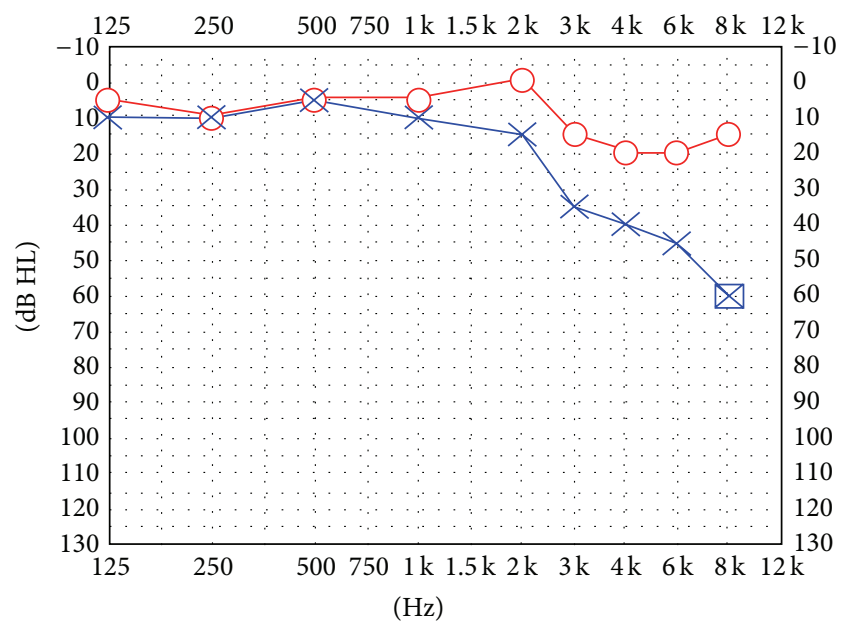

(b)

FIgURE 1: Pure tone audiogram at presentation (a) and at 3-month follow-up (b).

The patient was referred for vestibular testing and an MRI. Video head impulse testing showed a complete loss of left-sided semicircular canal function (Figure 2) and a dynamic visual acuity (DVA) test was highly pathological for the left and marginally pathological for the right side. Sacculus testing with cVEMPs was borderline pathological on the left, and utriculus testing with oVEMPs showed a 100\% loss on the left. Caloric testing showed a $77 \%$ asymmetry with feeble responses to cold water and no response to warm water.

The MRI of the petrous bone (Figure 3) showed normal anatomy, but with high intensity signal on unenhanced $\mathrm{T} 1 \mathrm{in}$ both the cochlea and vestibulum, consistent with a left sided ILH.

The patient was asked to stop his aspirin and observed. Now 6 months after initial presentation his vestibular loss is well compensated and his DVA and video head impulse tests have improved, though they are still pathological. Furthermore his hearing has partially recovered in the lower frequencies (Figures 1 and 2). Follow-up MRI also shows resorption of the blood (Figure 3) with no residual in the cochlea but some in the vestibule.

\section{Discussion}

Overall, the vestibular sense is not well known by the general public (indeed, it would be the famous 6th sense) and patients often use a variety of terms when trying to describe their vestibular symptoms. This coupled with the fact that many patients experience excellent compensation in a matter of days to weeks means that a significant vestibular dysfunction may only be apparent with vestibular testing. These need not be with advanced equipment in specialized centres as simple bedside tests are reliable indicators of vestibular dysfunction [19]. Most reports in the literature only use caloric testing to evaluate vestibular function whereas today it is possible to examine all 5 components of the vestibular system, namely, the three semicircular canals, utriculus, and sacculus.

Hearing loss is a far better understood problem by the general public, and indeed our patient's main complaint was of this, combined with tinnitus. Tinnitus can sometimes be the only symptom of a high frequency hearing loss, and many patients are astonished to see how poorly they hear at high frequencies. The acuter and more asymmetrical the hearing loss the more noticeable for the patient and the more likely they are to present for investigation.

In situations of both cochlear and vestibular dysfunction, careful history taking and examination can often elucidate the cause. Important differentials include infectious, vascular [20, 21], traumatic, neoplastic [22], iatrogenic (surgical [23] or drug-related [21]), and neurological causes of dysfunction.

Controversy still exists however as to when a patient should be referred for imaging [24], and often a diagnosis of "idiopathic" hearing or vestibular loss is made, without imaging to rule out other causes. Clearly in cases of suspected stroke, imaging is mandatory, but, for example, our patient only was referred to hospital and indeed underwent imaging some weeks after his initial symptoms.

In MRI, the labyrinthine endolymph has essentially the same signal intensity as cerebral spinal fluid (CSF) in both $\mathrm{T} 1$ and T2 sequences. (Perilymph has almost the same electrolyte composition as CSF although, higher protein whereas endolymph has the same protein concentration but higher potassium and lower sodium [25].) T1 hyperintense signals can in theory result from fat, protein, or blood [23]. Fat in the labyrinth is only seen in very rare lipomas. Protein in the perilymphatic fluid can be seen in middle ear infections or bacterial labyrinthitis. Careful radiological examination, especially with the use of 3D-Flair sequences [26], can effectively differentiate between ILH, acute inflammation and breakdown of the blood-labyrinth barrier [27] without the need for histological confirmation. However, as yet, the prognostic or therapeutic relevance of these findings is unclear.

To date, nearly all cases reported in the literature have permanent dysfunction following ILH with MRI findings still apparent $>6$ months after the original presentation. Happily for our patient, we were able to document audiological, vestibular, and radiological improvements. 

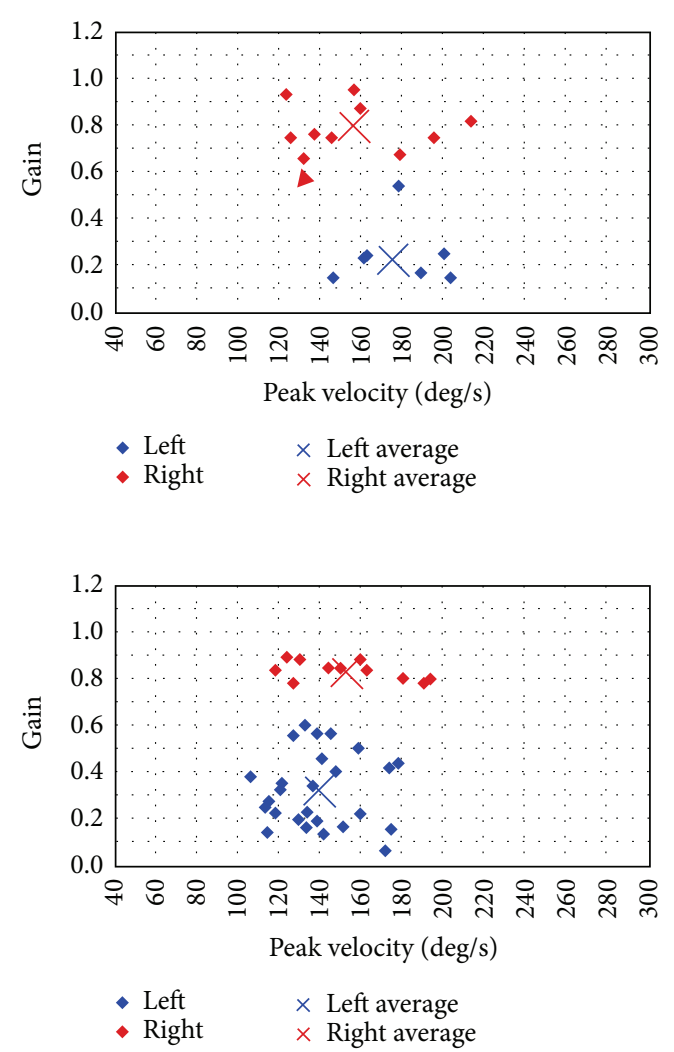

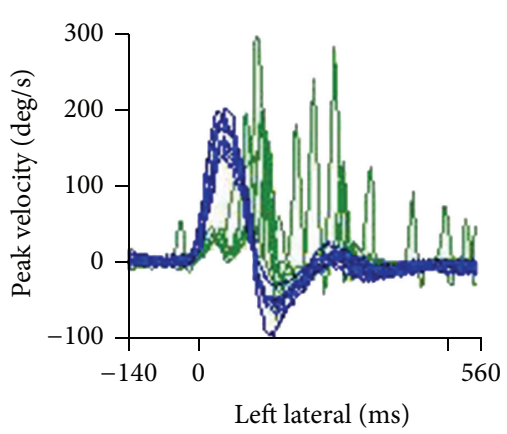

(a)
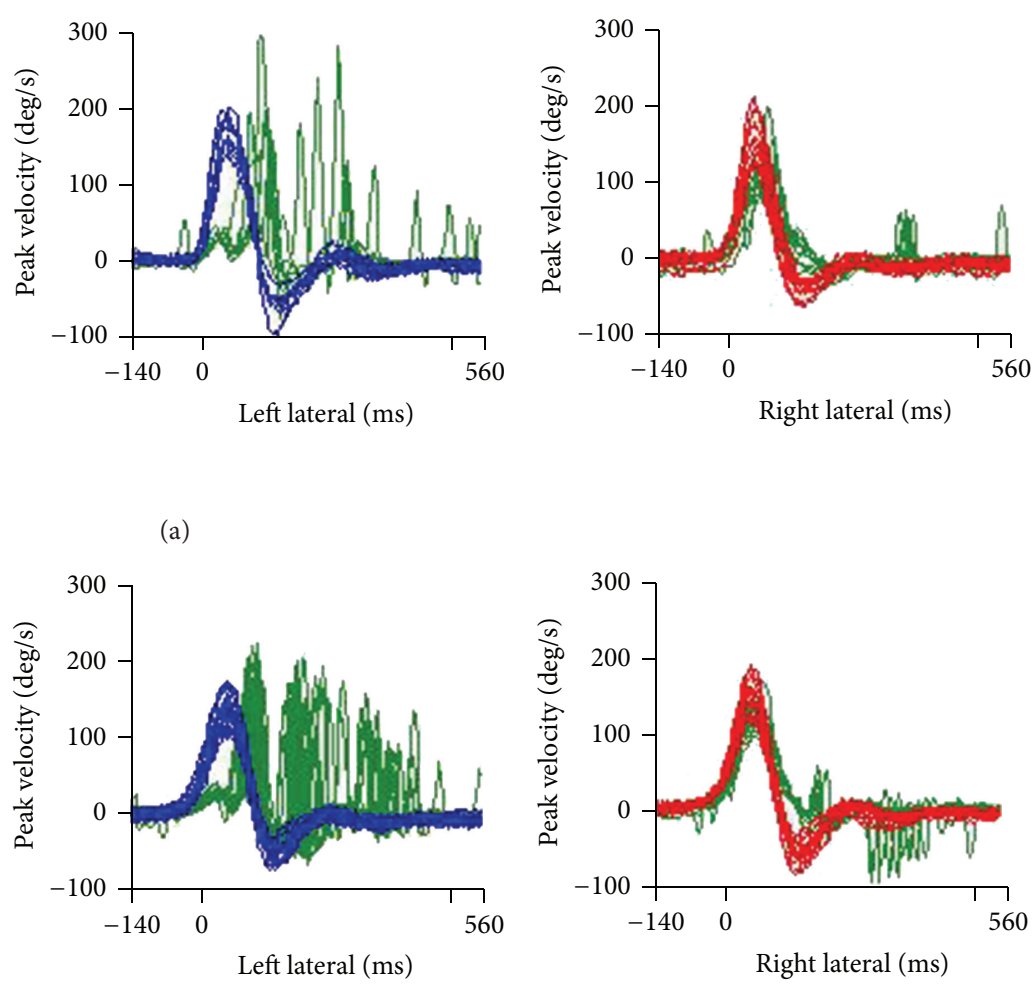

(b)

Figure 2: Video head impulse tests of the lateral semicircular canals at presentation (a) and at 3-month follow-up (b). Both show significant left sided correction saccades. DVA results however improved during follow-up.

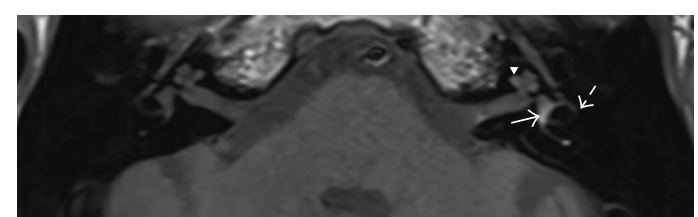

(a)

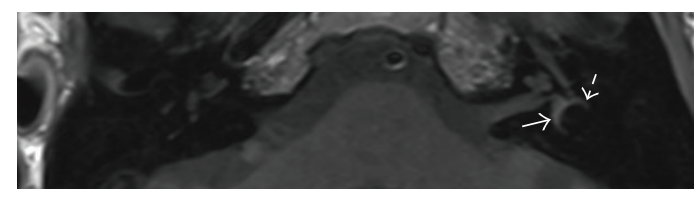

(b)

FIGURE 3: (a) T1 weighted native MRI of the petrous bone showing hemorrhage in the vestibule (arrow) and in the semicircular canals (partly shown, striped arrow) and slightly in the basal (not shown) and second turn (arrowhead) of the cochlea on the left. (b) 3month follow-up shows clear resolution of the hemorrhage. Only slight residual blood is visible in the vestibulum (arrow) and in the semicircular canals (partly shown, striped arrow).

\section{Conclusions}

ILH is a rare diagnosis best made on MRI. Most reports in the literature are of ILH secondary to clotting disorders, and indeed our patient had been taking low dose aspirin. It may be however that the phenomenon is underreported due to underimaging of patients and difficulties in accurate radiological diagnosis [28]. Histological confirmation is however no longer required. Furthermore, due to its rarity there is little consensus as to the optimal therapy, though treatment directed at any underlying cause (e.g., reversal of anticoagulation) would seem intuitively plausible. Certainly, efforts should be made to uncover occult bleeding diatheses. Of note, steroids and hyperbaric oxygen have not been shown to be superior to allowing the condition to run its natural course.

\section{Conflict of Interests}

The authors declare no conflict of interests.

\section{References}

[1] A. de Kleyn, "Sudden complete or partial loss of function of the octavus system in apparently normal persons," Acta OtoLaryngologica, vol. 32, no. 5-6, pp. 407-429, 1944.

[2] J. Kallinen, E. Laurikainen, P. Laippala, and R. Grénman, "Sudden deafness: a comparison of anticoagulant therapy and carbogen inhalation therapy," Annals of Otology, Rhinology and Laryngology, vol. 106, no. 1, pp. 22-26, 1997. 
[3] R. J. Stachler, S. S. Chandrasekhar, S. M. Archer et al., "Clinical practice guideline: sudden hearing loss," Otolaryngology-Head and Neck Surgery, vol. 146, no. 3, pp. S1-S35, 2012.

[4] R. Nosrati-Zarenoe, S. Arlinger, and E. Hultcrantz, "Idiopathic sudden sensorineural hearing loss: results drawn from the Swedish national database," Acta Oto-Laryngologica, vol. 127, no. 11, pp. 1168-1175, 2007.

[5] M. Suckfüll, "Perspectives on the pathophysiology and treatment of sudden idiopathic sensorineural hearing loss," Deutsches Ärzteblatt International, vol. 106, no. 41, pp. 669-676, 2009.

[6] G. B. Hughes, M. A. Freedman, T. J. Haberkamp, and M. E. Guay, "Sudden sensorineural hearing loss," Otolaryngologic Clinics of North America, vol. 29, no. 3, pp. 393-405, 1996.

[7] A. E. Conlin and L. S. Parnes, "Treatment of sudden sensorineural hearing loss I. A systematic review," Archives of Otolaryngology - Head and Neck Surgery, vol. 133, no. 6, pp. 573581, 2007.

[8] M. H. Bennett, T. Kertesz, M. Perleth, P. Yeung, and J. P. Lehm, "Hyperbaric oxygen for idiopathic sudden sensorineural hearing loss and tinnitus," Cochrane Database of Systematic Reviews, no. 10, Article ID CD004739, 2012.

[9] Z. Awad, C. Huins, and D. D. Pothier, "Antivirals for idiopathic sudden sensorineural hearing loss," Cochrane Database of Systematic Reviews, vol. 8, Article ID CD006987, 2012.

[10] N. O. Penido, O. L. M. Cruz, A. Zanoni, and D. P. Inoue, "Classification and hearing evolution of patients with sudden sensorineural hearing loss," Brazilian Journal of Medical and Biological Research, vol. 42, no. 8, pp. 712-716, 2009.

[11] P. Weinaug, "Spontaneous remissions in sudden hearing loss," HNO, vol. 32, no. 8, pp. 346-351, 1984.

[12] D. Egli Gallo, E. Khojasteh, M. Gloor, and S. C. Hegemann, "Effectiveness of systemic high-dose dexamethasone therapy for idiopathic sudden sensorineural hearing loss," Audiology \& Neurotology, vol. 18, no. 3, pp. 161-170, 2013.

[13] I. Braverman, J. Ben David, and A. Shupak, "MTHFR polymorphism: associated intralabyrinthine hemorrhage," Otolaryngology-Head and Neck Surgery, vol. 141, no. 4, pp. 541-542, 2009.

[14] P. R. Sabatini and J. W. Kutz Jr., "Radiology quiz case 1. Intralabyrinthine hemorrhage," Archives of OtolaryngologyHead and Neck Surgery, vol. 135, no. 6, pp. 612-614, 2009.

[15] W. M. Rosado Jr. and E. Palacios, "Sudden onset of sensorineural hearing loss secondary to intralabyrinthine hemorrhage: MRI findings," Ear, Nose and Throat Journal, vol. 87, no. 3, pp. 130-131, 2008.

[16] C. Toyama, C. J. da Silva, F. T. Braga, and R. Brito, "Intralabyrinthine hemorrhage associated with superficial siderosis of the central nervous system," Otology and Neurotology, vol. 30, no. 1, pp. 121-122, 2009.

[17] J. Jagannathan, J. A. Butman, R. R. Lonser et al., "Endolymphatic sac tumor demonstrated by intralabyrinthine hemorrhage. Case report," Journal of Neurosurgery, vol. 107, no. 2, pp. 421-425, 2007.

[18] B. B. Kuzma and J. M. Goodman, "Hemorrhagic lesions causing acute sensorineural hearing loss," Surgical Neurology, vol. 49, no. 6, pp. 660-661, 1998.

[19] C. Rohrmeier, O. Richter, M. Schneider et al., "Triple test as predictive screen for unilateral weakness on caloric testing in routine practice," Otology \& Neurotology, vol. 34, no. 2, pp. 297303, 2013.
[20] R. Salomone, T. A. A. Abu, A. G. Chaves, M. C. C. Bocalini, A. D. O. Vicente, and P. E. Riskalla, "Sudden hearing loss caused by labyrinthine hemorrhage," Brazilian Journal of Otorhinolaryngology, vol. 74, no. 5, pp. 776-779, 2008.

[21] M. Kothari, F. Knopp, S. Jonas, and D. Levine, "Presumed vestibular hemorrhage secondary to warfarin," Neuroradiology, vol. 37, no. 4, pp. 324-325, 1995.

[22] H. F. Schuknecht, M. Igarashi, and W. D. Chasin, "Inner ear hemorrhage in leukemia. A case report," The Laryngoscope, vol. 75, pp. 662-668, 1965.

[23] J. L. Weissman, H. D. Curtin, B. E. Hirsch, and W. L. Hirsch Jr., "High signal from the otic labyrinth on unenhanced magnetic resonance imaging," American Journal of Neuroradiology, vol. 13, no. 4, pp. 1183-1187, 1992.

[24] F. Craighero, J. W. Casselman, M. M. Safronova, B. de Foer, J. Delanote, and E. F. Officiers, "Sudden onset vertigo: imaging work-up," Journal de Radiologie, vol. 92, no. 11, pp. 972-986, 2011.

[25] P. Wangemann and J. Schacht, "Homeostatic mechanisms in the cochlea," in The Cochlea, P. Dallos, A. Popper, and R. Fay, Eds., pp. 130-185, Springer, New York, NY, USA, 1996.

[26] S. Berrettini, V. Seccia, S. Fortunato et al., "Analysis of the 3dimensional fluid-attenuated inversion-recovery (3D-FLAIR) sequence in idiopathic sudden sensorineural hearing loss," JAMA Otolaryngology - Head \& Neck Surgery, vol. 139, no. 5, pp. 456-464, 2013.

[27] H. Y. Lee, S. Y. Jung, M. S. Park, S. G. Yeo, S. Y. Lee, and S. K. Lee, "Feasibility of three-dimensional fluid-attenuated inversion recovery magnetic resonance imaging as a prognostic factor in patients with sudden hearing loss," European Archives of Oto-Rhino-Laryngology, vol. 269, pp. 1885-1891, 2012.

[28] F. Dubrulle, R. Kohler, C. Vincent, P. Puech, and O. Ernst, "Differential diagnosis and prognosis of T1-weighted postgadolinium intralabyrinthine hyperintensities," European Radiology, vol. 20, no. 11, pp. 2628-2636, 2010. 


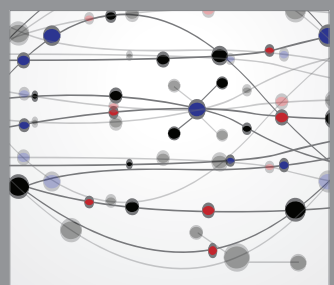

The Scientific World Journal
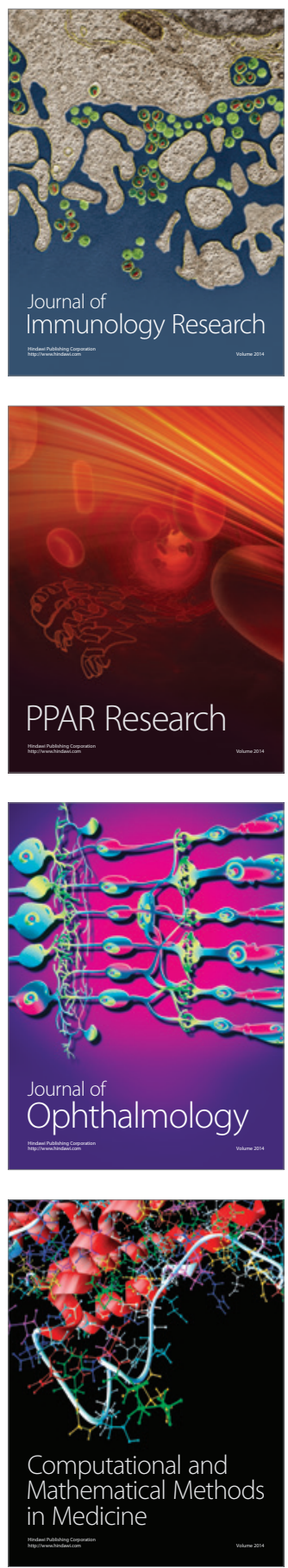

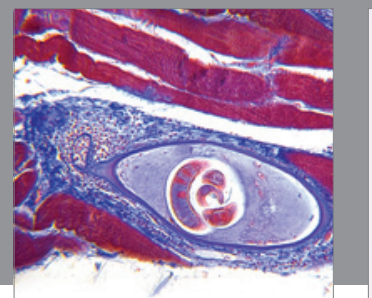

Gastroenterology

Research and Practice
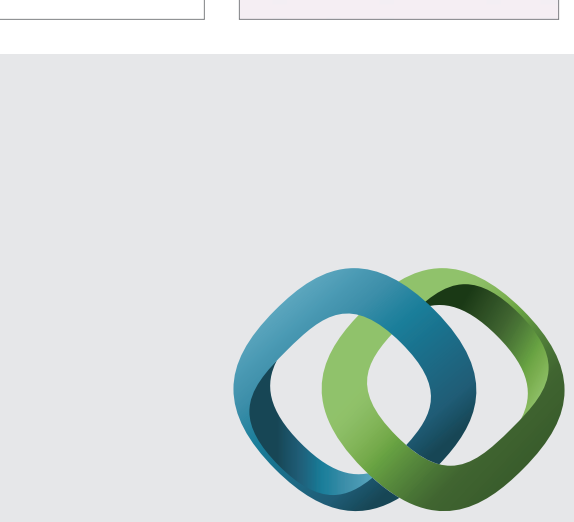

\section{Hindawi}

Submit your manuscripts at

http://www.hindawi.com
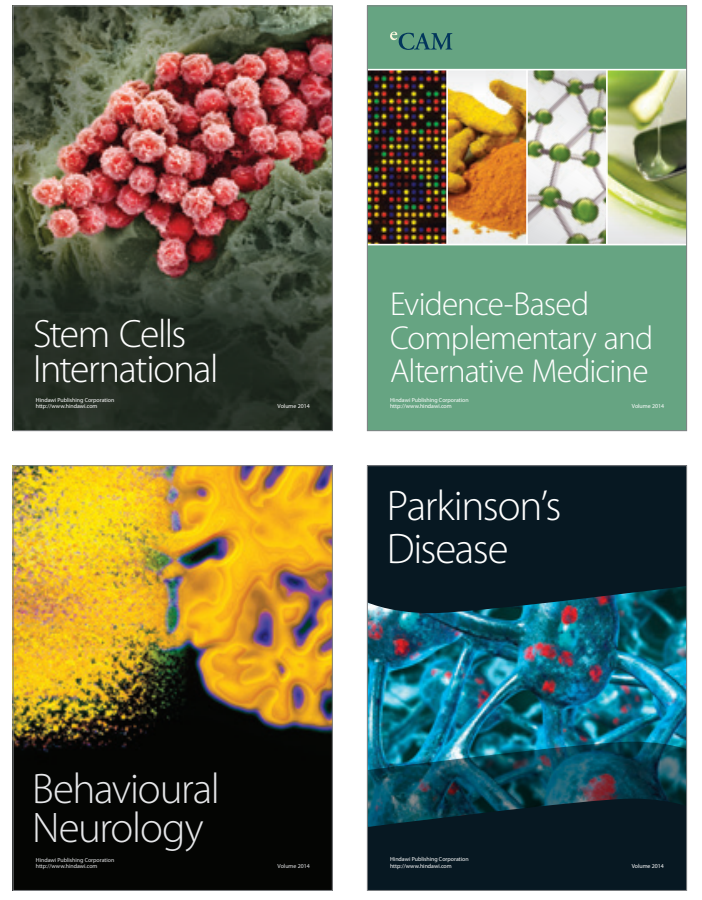
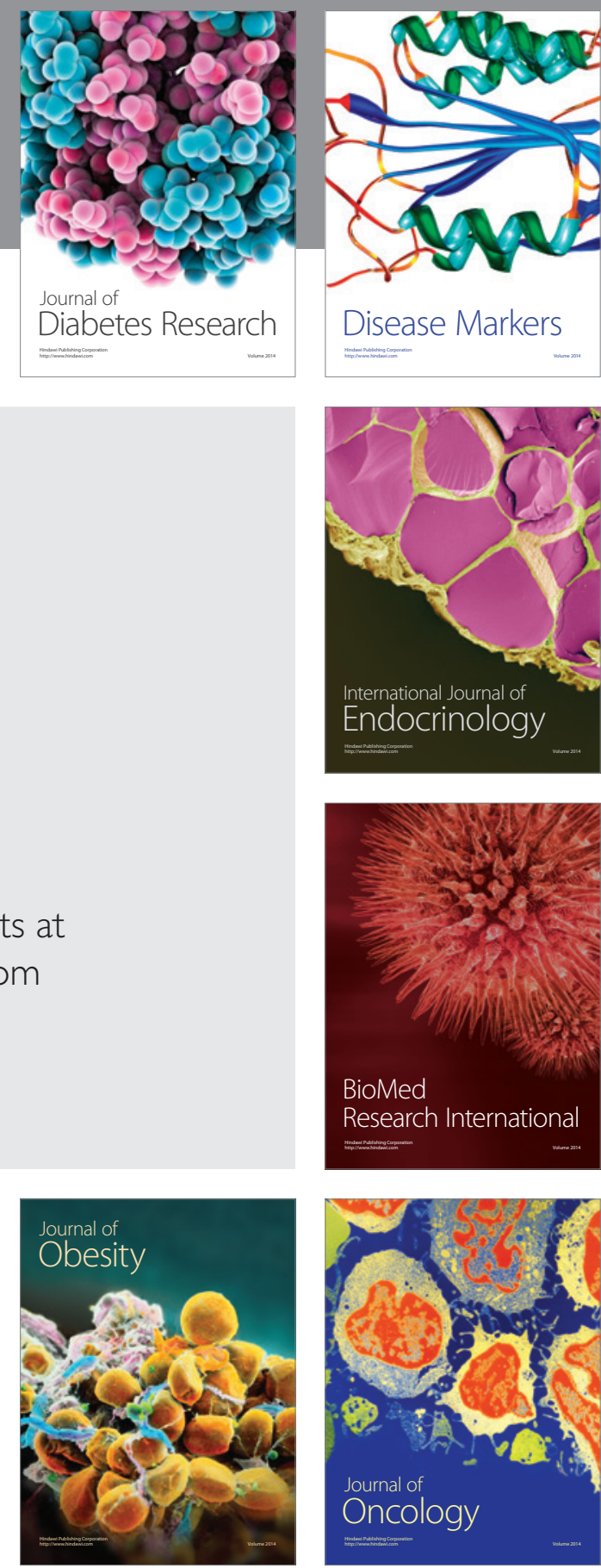

Disease Markers
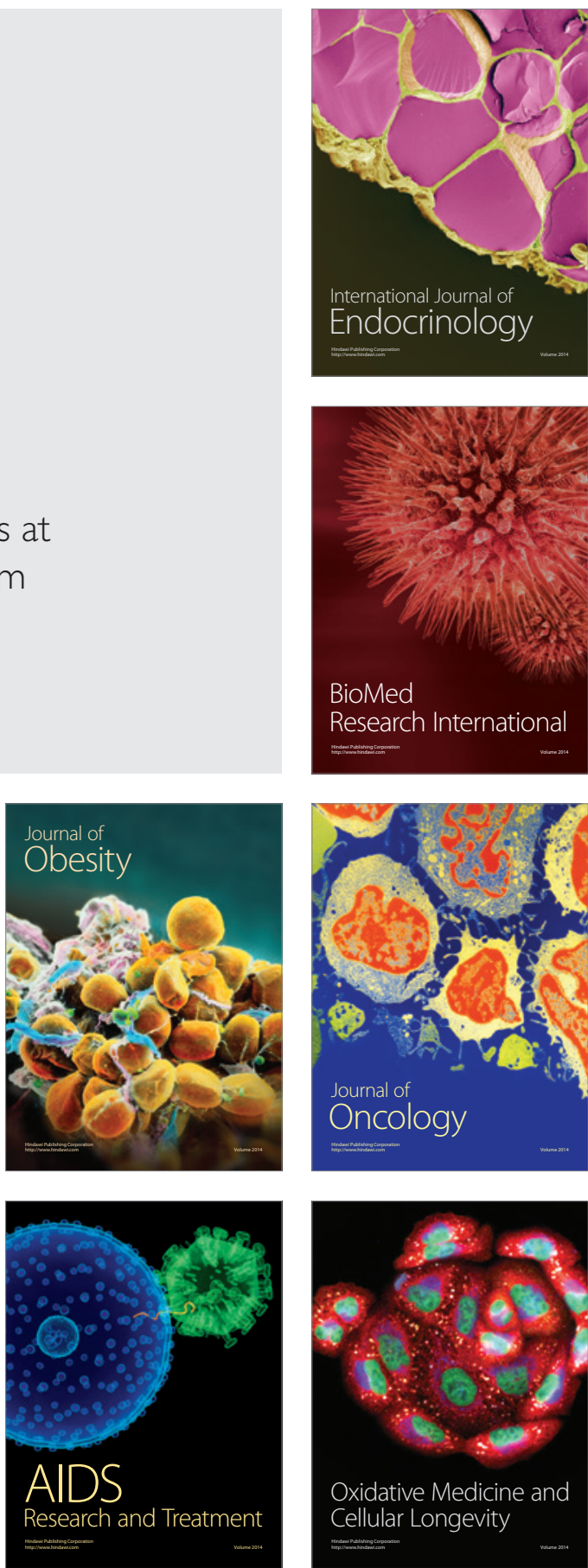